\title{
FUNGOS MICORRÍZICOS ARBUSCULARES EM RIZOSFERAS DE PLANTAS DO LITORAL ARENOSO DO PARQUE ESTADUAL DA ILHA DO CARDOSO, SP, BRASIL. 2.
}

\author{
Sandra F. B. Trufem ${ }^{1}$ \\ Sandra M.M. Malatinszky ${ }^{1,2}$ \\ Helena S. Otomo ${ }^{1,3}$
}

Recebido em 7.12.92. Aceito em 05.9.94

\begin{abstract}
RESUMO - (Fungos micorrízicos arbusculares em rizosferas de plantas do litoral arenoso do Parque Estadual da Ilha do Cardoso, SP, Brasil. 2). De março/1988 a julho/1990, em 14 ocasiões, foram coletadas 410 amostras de solo de rizosfera de plantas do litoral arenoso da Ilha do Cardoso, SP, Brasil, com finalidade de se conhecer a micota de fMA. Foram investigadas as rizosferas das plantas: Baccharis trimera DC., Blutaparon portulacoides (St. Hil.) Mears, Dalbergia hecastaphylla (L.) Taub., Hydrocotyle bonariensis Lam., Ipomoea pes-caprae (L.) Swett e Polygala cyparisseas St. Hil. \& Moq. Verificaram-se 24 espécies de fMA. Os resultados obtidos permitiram constatar que: a) há tendência em ter-se aumento no número de esporos no solo com o aumento da temperatura, precipitação e insolação; b) diferentes espécies de fMA ocorrem em diferentes espécies de plantas hospedeiras, sugerindo a existência de especificidade ecológica; c) há maior abundância de esporos das espécies de Acaulospora, Gigaspora e Scutellospora sobre as de Glomus. Não se verificou relação entre a fenologia das plantas hospedeiras, o número de esporos no solo e a porcentagem de colonização das raízes.
\end{abstract}

Palavras-chave: fungos MA, litoral arenoso, ecologia.

ABSTRACT - (Arbuscular mycorrhizal fungi in plant rhizospheres from sandy soil of Parque Estadual da Ilha do Cardoso, SP, Brazil. 2). From March/1988 to July/1990, in 14 opportunities, it was collected 410 soil samples of rhizospheres of native plants from sandy soils of Ilha do Cardoso, SP, Brazil to investigate the occurrence of AM fungi. The studied plants were: Baccharis trimera DC., Blutaparon portulacoides (St. Hil.) Mears, Dalbergia hecastaphylla (L.) Taub., Hydrocotyle bonariensis Lam., Ipomoea pes-caprae (L.) Swett and Polygala cyparisseas St. Hil. It was observed 24 species of AM fungi. The results showed: a) a tendency to increase the number of spores in the soil with the increase of the temperature, rainfall and sunlight; b) different species of AM fungi occurred in different species of hosts, suggesting ecological specificity of AM fungi; c)

\footnotetext{
' Instituto de Botânica, Caixa Postal 4005, 01061-970, São Paulo-SP, Brasil.

${ }^{2}$ Bolsa de Iniciação Científica, CNPq, processo n. ${ }^{0} 804$ 928/87-8.

${ }^{3}$ Bolsa de Aperfeiçoamento, CNPq, processo n. ${ }^{0} 820$ 655/86-4.
} 
it was observed higher abundance of spores of Acaulospora, Gigaspora and Scutellospora over spores of Glomus and Sclerocystis. It was not observed relatonships between the number of spores in the soil, taxa of colonization of the roots and the fenology of hosts plants.

Key words: AM fungi, sandy soil, ecology.

\section{Introdução}

Os fungos micorrízios arbusculares (fMA) são organismos simbiotróficos obrigatórios da maioria das plantas vasculares, constituindo exceção a sua ausência (Gerdemann 1968, Janos 1980). A simbiose fica estabelecida quando o fungo cede à planta hospedeira água e sais mineirais retirados do solo e recebe dela produtos da fotossíntese (Cabala Rosand \& Dias 1986, Siqueira \& Franco 1988).

Trabalhos diversos relatam a participação decisiva e positiva dos fMA na fixação de dunas (Koske 1981, Koske \& Polson 1984), na recomposição de vegetação de áreas sujeitas a secas (Allen \& Boosalis 1983) ou submetidas a impactos ambientais diversos, tais como chuvas ácidas (Killham \& Firestone 1983), depósito de metais pasados (Gildon \& Tinker 1983a, 1983b), deslizamentos (Sylvia \& Will 1988), áreas de mineração (Daft \& Nicolson 1974), entre outros

$\mathrm{O}$ estudo de fMA sob vegetação nativa em áreas preservadas fornece dados sobre a biologia e a ecologia desses organismos em áreas de menor interferência antrópica, oferecendo subsídios para seu manejo em outras situações.

Constituem objetivos deste trabalho o relato da ocorrência de fMA em plantas nativas do litoral arenoso da Ilha do Cardoso, com algumas considerações sobre apectos ecológicos desses organismos.

\section{Material e métodos}

De março/1988 a julho/1990, em 14 oportunidades, foram coletadas 82 amostras compostas de solo de rizosfera de plantas da Praia de Fora, no Parque Estadual da Il ha do Cardoso ( $25^{\circ} 05^{\prime}$ e $25^{\circ} 15^{\prime} \mathrm{S}$ e $\left.47^{0} 53^{\prime} \mathrm{e} 48^{\circ} 06^{\prime} \mathrm{W}\right)$. Cada cinco amostras de solo de rizosfera provenientes de cinco indivíduos distintos, porém de mesma espécie, constituiram uma mostra composta. Assim, foram investigadas 410 rizosferas. As plantas que tiveram suas rizosferas estudadas foram: Baccharis trimera DC., Blutaparon portulacoides (St. Hil.) Mears, Dalbergia hecastaphylla (L.) Taub., Hydrocotyle bonariensis Lam., Ipomoea pes-caprae (L.) Sweet e Polygala cyparisseas St. Hil. \& Moq. Estas plantas foram selecionadas por serem abundantes na região estudada.

Para o isolamento dos esporos de fMA, o solo coletado foi preparado pela técnica da lavagem, decantação e peneiramento por via úmida (Gerdemann \& Nicolson 1963). A colonização das raízes foi evidenciada com fucsina ácida (Kormanik et al. 1980).

Por ocasião das coletas, foram anotados dados fenológicos da planta hospedeira para eventuais relações com a ocorrência de esporos no solo e/ou porcentagem de colonização das raízes. 
Dados climatológicos foram obtidos junto ao Instituto Oceonográfico da Universidade de São Paulo, que mantém estação no município de Cananéia.

Amostras de solo foram encaminhadas ao Instituto Agronômico, Campinas, SP, para análise dos elementos químicos.

\section{Resultados e discussão}

Foram verificadas 24 espécies de fMA, a saber: Acauospora laevis Gerd. \& Trappe, A. scrobiculata Trappe, A. spinosa Walker \& Trappe, A. tuberculata Janos \& Trappe, Acaulospora sp., Gigaspora decipiens Hall \& Abbott, G. gigantea (Nicol. \& Gerd.) Walker \& Sanders, G. ramisporophora Spain, Sieverding \& Schenck, Glomus aggregatum Schenck \& Smith emend. Koske, G. etunicatum Becker \& Gerd., G. constrictum Trappe, G. fasciculatum (Thaxter) Gerd. \& Trappe emend. Walker \& Koske, G. globiferum Koste \& Walker, G. monosporum Gerd. \& Trappe, G. tenue (Greenall) Hall,Sclerocystis sinuosa Gerd. \& Bakshi,Scutellospora calospora (Nicol. \& Gerd.) Walker \& Sandes, S. coralloides (Trappe, Gerd. \& Ho) Walker \& Sanders, S. fulgida Koske \& Walker, S. gilmorei (Trappe \& Gerd.) Walker \& Sanders, G. gregaria (Schenck \& Nicol.) Walker \& Sanders, S. persica (Koske \& Walker) Walker \& Sanders, S. verrucosa (Koske \& Walker) Walker \& Sandrers e Scutellospora sp.

À exceção de $G$. aggregatum, $G$. constrictum e $G$. ramisporophora, todas as demais espécies foram descritas e comentadas em trabalhos anteriores publicados no Brasil (Trufem 1988, Trufem et al. 1989), assim, aqui são apresentados esses dados apenas para os táxons verificados pela primeira vez na Ilha do Cardoso.

Glomus aggregatum Schenck \& Smith emend. Koske, Mycologia 77:619-630. 1985.

Esporos em grupos de até 20 indivíduos, sem perídio; esporos amarelo-escuros a castanhos, esféricos, 60-65 $\mu \mathrm{m}$ diâm. a levemente alongados, 60-68 (-80) x 50-60 $\mu \mathrm{m}$ diâm., parede em um grupo: parede 1 amarela a castanha, laminada, 3,0-5,0 $\mu \mathrm{m}$ espessa; hifa de sustentação reta, castanho-amarela, mais clara que o esporo, sem septo, 8,0-10 (-15) $\mu \mathrm{m}$ diâm., parede afinando-se gradualmente ao longo da hifa de sustentação, até $2,0 \mu \mathrm{m}$ espessa.

Esporos verificados em rizosferas de B. trimera (XII/1989).

Comentários: boa correspondência do material estudado com os dados fornecidos na descrição de Koske (1985).

Glomus constrictum Trappe, Mycotaxon 6:359-366.1977.

Esporos isolados no solo ou, mais freqüentemente, em grupos de até 100 indivíduos, sem perídio; esporos catanho-avermelhados, brilhantes, esféricos, (110-) 123-155 (-170) $\mu \mathrm{m}$ diâm.; parede em um grupo: parede 1 catanho-avermelhada, laminada, 5,0-14 $\mu$ m espessa; hifa de sustentação castanha significativamente mais clara que o esporo, geralmente fortemente recurvada, com constricção nítida junto ao 
ponto de inserção, onde mede até $10 \mu \mathrm{m}$ diâm., parede até $4,0 \mu \mathrm{m}$ espessa; hifa de

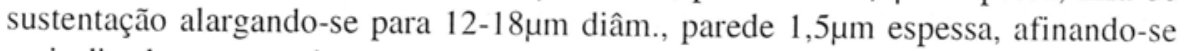
mais distalmente, sendo comum a ocorrência de bifurcação.

Esporos verificados em rizosferas de B. trimera (IX/1988; X/1988; V/1989; VII/1989); D. hecastaphylla (IV/1988); H. bonariensis (IV/1988; V/1989) e P.cyparisseas (III/1988; IV/1988).

Comentários: boa correspondência do material estudado com os dados da descrição original (Trappe 1977).

Gigaspora ramisporophora Spain, Sieverding \& Schenck, Mycotaxon 34:667677.1989.

Esporos isolados no solo, amarelo-claros com leves tons esverdeados quando jovens, passando a castanho claros e mesmo castanho-dourados com a idade, esféri-

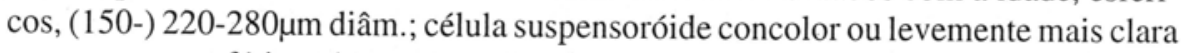
que o esporo, esférica a levemente clavada, 32-41 $\mu \mathrm{m}$ diâm., parede até 8,0 0 m espessa junto à inserção no esporo, até $4,0 \mu \mathrm{m}$ espessa nas laterais; hifa de sustentação concolor à célula suspensoróide, septada ou não, 8,0-12 $\mu \mathrm{m}$ diâm., $50 \mu \mathrm{m}$ compr. ou mais, sendo comum sua ramificação; parede do esporo em um grupo: parede 1 hialina, unitária, até $1,5 \mu \mathrm{m}$ espessa, à qual adere a parede 2 , amarela a castanha, nitidamente laminada, tanto mais espessa quando mais velho o esporo, $5,0-15 \mu \mathrm{m}$ espessa; parede 3 aderida e concolor à parede 2, com série de projeções voltadas para o interior do esporo; projeções verrucosas ou cônicas, com ápice arrendondado, até 3,0 $\mu \mathrm{m}$ alt., até $1,8 \mu \mathrm{m}$ larg. na base; células auxiliares não observadas.

Esporos verificados em rizosferas de B. trimera., B. portulacoides e $P$. cyparisseas em todas as ocasiões de coletas (III/1988; IV/1988; V/1988; VII/1988; VIII/1988; IX/1988; X/1988; XI/1988; XII/1988; I/1989; II/1989; III/1989; V/1989 e VII/1989). Em I. pes-caprae a espécie ocorreu apenas na coleta de I/1989.

Comentários: boa concordância com a descrição original (Spain et al. 1989).

Os dados climatológicos (tab.1) permitem constatar que agosto/1988 foi o mês mais seco e janeiro/1989, o mais chuvoso (10,1 e 459,3mm de precipitação ao mês, respectivamente), sendo julho/1988 o mês mais frio e fevereiro/1989, o mais quente ( 16,2 e $26,5^{\circ} \mathrm{C}$ de média das temperaturas mensais, respectivamente). Março/1988 foi o mês com o maior número de horas de insolação e setembro/1988, com menor (188,6 e 62,9 horas de insolação ao mês, respectivamente). Esses dados vêm de encontro aos apresentados por Pfeiffer (1981/1982),que identifica o clima da região, segundo classificação de Koeppen, como do tipo Cfa, mesotérmico úmido, sem estação seca.

A análise da tab. 2 permite verificar que $P$. cyparisseas foi a planta cuja rizosfera apresentou maior abundância de esporos, com a média geral de 142 esporos $/ 100 \mathrm{~g}$ de solo. Esses resultados confirmam trabalho anterior (Trufem 1990) que constatou que 


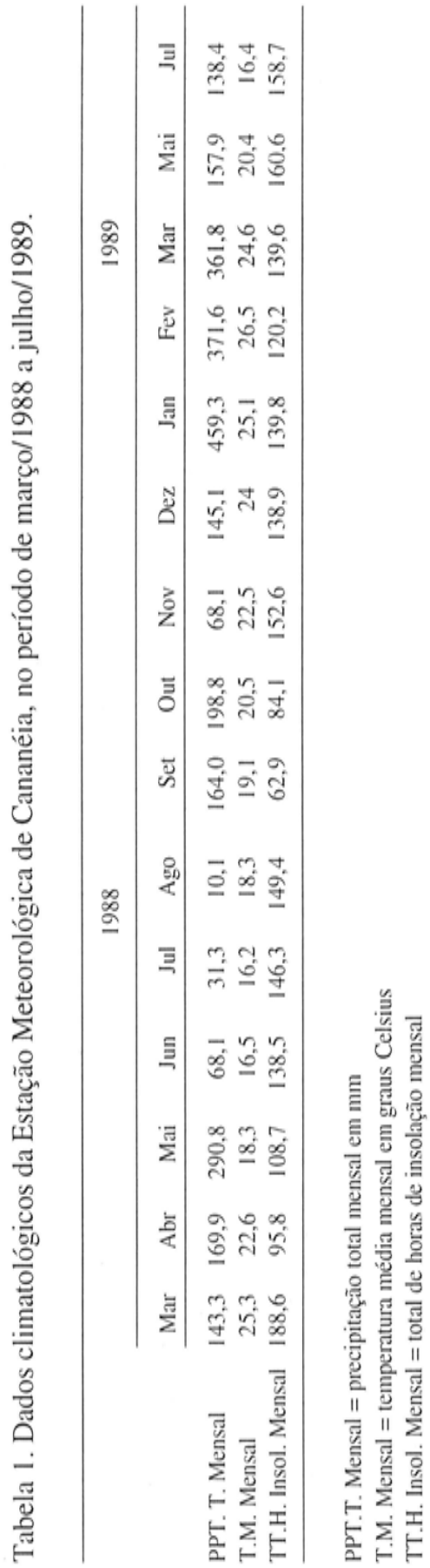


essa mesma planta, para a mesma área, foi a que apresentou maior quantidade de esporos de fMA em sua rizosfera. A literatura (Gerdemann 1968, Mosse et al. 1981), embora comente que há plantas, como as Compositae que são micotróficas, não estabelece situação para Polygalaceae, caso de $P$. cyparisseas.

Tabela 2. Número de esporos de $\mathrm{fMA} / 100 \mathrm{~g}$ de solo nas rizosferas das plantas estudadas em função das épocas de coletas.

\begin{tabular}{|c|c|c|c|c|c|c|c|c|}
\hline & 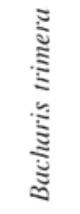 & 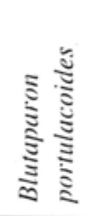 & 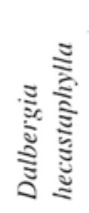 & 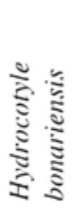 & 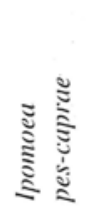 & 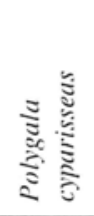 & Tot. & Med. \\
\hline Mar & $\mathrm{NC}$ & 2 & 8 & 2 & 0 & 515 & 527 & 105 \\
\hline Abr & $\mathrm{NC}$ & 0 & 19 & 20 & 0 & 526 & 565 & 113 \\
\hline Mai & 17 & 3 & 20 & 29 & 0 & 534 & 603 & 101 \\
\hline Jun & 42 & 0 & 0 & 11 & 5 & 23 & 81 & 14 \\
\hline Ago & 97 & 2 & 102 & 19 & 0 & 19 & 300 & 50 \\
\hline Set & 154 & 23 & 123 & 0 & 0 & 0 & 177 & 30 \\
\hline Out & 43 & 0 & 20 & 0 & 3 & 25 & 91 & 15 \\
\hline Nov & 108 & 0 & 52 & 0 & 0 & 6 & 166 & 28 \\
\hline Dez & 71 & 3 & 70 & 0 & 0 & 12 & 156 & 26 \\
\hline Jan & 231 & 3 & 46 & 0 & 15 & 6 & 301 & 50 \\
\hline Fev & 108 & 6 & 108 & 77 & 77 & 3 & 379 & 63 \\
\hline Mar & 54 & 0 & 154 & 0 & 3 & 31 & 242 & 40 \\
\hline Mai & 154 & 0 & 23 & 46 & 2 & 62 & 287 & 48 \\
\hline Jul & 231 & 0 & 100 & 17 & 2 & 231 & 581 & 97 \\
\hline NTE & 1310 & 42 & 845 & 221 & 107 & 1993 & 4518 & - \\
\hline NME & 109 & 3 & 60 & 16 & 8 & 142 & - & 56 \\
\hline
\end{tabular}

NTE $=n$. total de esporos

$\mathrm{NME}=$ média do $\mathrm{n}$. de esporos fMA $/ 100 \mathrm{~g}$ de solo

Percebe-se que B. portulacoides, I. pes-caprae e H. bonariensis, espécies que normalmente se situam na linha de frente do litoral arenoso, foram as plantas hospedeiras que apresentaram as menores médias de esporos em suas rizosferas ( 3,8 e 16 esporos/100g de solo, respectivamente). Esses dados confirmam propostas de literatura que mencionam que as plantas ditas verdadeiramente pioneiras ou colonizadoras são não micotróficas (Reeves et al. 1979).

Por outro lado, $D$. hecastaphylla, B. trimera e $P$. cyparisseas apresentaram os maiores números médios de esporos de fMA/100g de solo (60, 109 e 142, respectivamente). Não apenas coincidentemente, essas plantas ocorrem em áreas mais interiores ao litoral, não estando, portanto, submetidas às condições tão estressantes quanto as de B. portulacoides, I. pes-caprae e H. bonariensis. Logo, não são efetivamente consideradas como pioneiras, exibindo já a micotrofia. 
Analizando-se as tab. 1 e 2 simultaneamente, nota-se que os meses que apresentaram maior média em relação ao número de esporos/100g de solo, foram os de abril/ 1988 (113 esporos) e março/1988 (105 esporos), enquanto que as menores médias ocorreram nos meses de julho/1988 (14 esporos) e outubro/1988 (15 esporos). Buscando-se relação com os dados climatológicos, percebe-se a menor média no número de esporos em épocas próximas àquelas em que as precipitações foram menores, as temperaturas foram mais baixas e houve menor número de horas de insolação. Já as maiores médias no número de esporos aconteceram com o aumento da temperatura e o maior número de horas de insolação.

Para o aumento do número de esporos os presentes dados mostram maior relação com as horas de insolação do que com a precipitação e a temperatura. Assim, com o aumento do número de horas de insolação, havia aumento no número de esporos e vice-versa.

A análise da tab. 3 demonstra que, de modo geral, não se observou colonização por fMA nas raízes das plantas hospedeiras estudadas. A exceção ocorreu nos meses de maio/1989 e julho/1989, ocasião em que a maioria apresentou colonização de até $80 \%$, exceto $I$. pes-caprae, que nunca apareceu colonizada. B. trimera foi a planta que com maior freqüência mostrou arbúsculos e vesículas em suas raízes, seguindo-se $H$. bonariensis. Confrontando-se esses resultados com o número total de esporos verificado na rizosfera de cada planta (tab. 2), não se encontrou relação entre o número de esporos no solo e a pocentagem de colonização das raízes. Assim, $P$. cyparisseas, que apresentou o maior número de esporos no solo, não foi a planta com maiores e nem mais constantes taxas de colonização. B. trimera apresentou o segundo número de esporos e as maiores e mais constantes taxas de colonização, apresentou baixo número de esporos. Os dados de número de esporos no solo e porcentagem de colonização das raízes são contraditórios em literatura, existindo autores que verificaram comportamentos paralelos entre os dois aspectos (Giovannetti, 1985; Sylvia \& Will, 1988) e inversos (Louis \& Lim 1987), e não relacionados (Anderson et al. 1983), dentre os quais nos alinhamos.

Tabela 3. Porcentagem de colonização das raízes das plantas hospedeiras estudadas em função das épocas de coletas.

\begin{tabular}{|c|c|c|c|c|c|c|c|c|c|c|c|c|c|c|}
\hline \multirow{2}{*}{$\begin{array}{l}\text { planta } \\
\text { hospedeira }\end{array}$} & \multicolumn{8}{|c|}{1988} & \multicolumn{6}{|c|}{1989} \\
\hline & Mar & Abr & Mai & Jun & Ago & Set & Out & Nov & Dez & Jan & Fev & Mar & Mai & Jul \\
\hline B. trimera & $\mathrm{NC}$ & $\mathrm{NC}$ & 50 & $\mathrm{NC}$ & 10 & - & 90 & 50 & - & - & - & - & - & 10 \\
\hline B. portulacoides & - & - & 90 & $\mathrm{NC}$ & - & - & - & - & - & - & - & 10 & - & - \\
\hline D. hecastaphylla & - & - & 80 & $\mathrm{NC}$ & 5 & - & - & - & - & - & - & - & - & - \\
\hline H. bonariensis & - & 5 & 80 & $\mathrm{NC}$ & 90 & 80 & - & - & - & - & - & - & - & - \\
\hline I. pes-caprae & - & - & - & $\mathrm{NC}$ & - & - & - & - & - & - & - & - & - & - \\
\hline P. cyparisseas & - & - & - & $\mathrm{NC}$ & 10 & 90 & - & - & - & - & - & - & - & - \\
\hline
\end{tabular}

$\mathrm{NC}=$ amostra não coletada

- = colonização não observada 
A tab. 4, quando analisada em conjunto com a tab. 3, não permite conclusões. Verificou-se aumento na porcentagem de colonização das raízes quando ocorreu floração para $H$. bonariensis, porém, plantas que floresceram o ano todo apresenteram porcentagens diferentes de colonização ( $P$. cyparisseas, com 10 a 90\%), o mesmo tendo se verificado para plantas que permaneceram no estado vegetativo o ano todo (D. hecastaphylla, 5 a 80\%). De modo tênue, pode-se notar que, quando começou a declinar a temperatura (maio-julho, tab. 1) as raízes se apresentaram colonizadas em maior porcentagem. Esses dados são concordantes com os de Sylvia \& Will (1988), que verificaram aumento no número de esporos no solo com esse tipo de variação ambiental.

Tabela 4. Fenologia das plantas hospedeiras estudadas em função das épocas de coletas

1988

1989

planta

hospedeira Mar Abr Mai Jun Jul Ago Set Out Nov Dez Jan Fev Mar Mai Jul

\begin{tabular}{lrrrrrrrrrrrrrrr}
\hline B. trimera & $\mathrm{NC}$ & $\mathrm{NC}$ & / & $\mathrm{NC}$ & $/$ & $*$ & $/$ & $/$ & $/$ & $*$ & $/$ & $*$ & $*$ & $/$ & / \\
B. portulacoides & $/$ & $/$ & $/$ & $\mathrm{NC}$ & $/$ & $*$ & $/$ & $/$ & $/$ & $*$ & $*$ & $*$ & $*$ & $*$ & $/$ \\
D. hecastaphylla & $* 0$ & $/$ & $/$ & $\mathrm{NC}$ & $/$ & $/$ & $/$ & $/$ & $/$ & $/$ & $/$ & $/$ & $/$ & $/$ & $/$ \\
H. bonariensis & $*$ & $/$ & $/$ & $\mathrm{NC}$ & $*$ & $*$ & $*$ & $*$ & $*$ & $*$ & $*$ & $*$ & $*$ & $*$ & $*$ \\
I. pes-caprae & $* 0$ & $* 0$ & $* 0$ & $\mathrm{NC}$ & $/$ & $/$ & $/$ & $*$ & $*$ & $*$ & $*$ & $*$ & $* 0$ & $* 0$ & $/$ \\
P. cyparisseas & $*$ & $*$ & $*$ & $\mathrm{NC}$ & $*$ & $*$ & $*$ & $*$ & $*$ & $*$ & $*$ & $*$ & $*$ & $*$ & $*$ \\
\hline
\end{tabular}

$\mathrm{NC}=$ não coletada

I = estado vegetativo

* = em floração

$0=$ com frutos $/$ sementes

No tocante à distribuição de fMA em função da rizosfera da planta hospedeira, a tab. 5 demostra que $G$. gigantea, $S$. coralloides e Scutellospora sp. foram espécies que ocorreram em todas as rizosferas, enquanto as demais ocorreram em cinco, quatro, três, duas ou apenas uma das espécies de planta hospedeira. Esses dados confirmam observações de Koske \& Halvorson (1981), Trufem \& Bononi (1985) e outros, que advogam preferências maiores ou mais acentuadas de fMA por determinadas plantas hospedeiras, sugerindo, portanto, a existência de "especificidade ecológica", proposta por McGonigle \& Fitter (1990).

No que se refere à diversidade de fMA em função da rizosfera da planta hospedeira (tab. 5), percebe-se que B. trimera foi a planta que comportou a maior diversidade de fMA, com 20 espécies, seguindo-se $P$. cyparisseas, com 17 espécies, D. hecastaphylla e H. bonariensis com dez, I. pes-caprae com sete e B. portulacoides com seis. A literatura (Koske, 1987) reporta até 14 espécies de fMA verificadas em uma mesma rizosfera (Uniola sp.). Mais recentemente, Trufem (1990) verificou 19 espécies de fMA em rizosfera de Diodia radula Cham, \& Schlecht. e de Smilax elastica Griseb., para o litoral arenoso da mesma Ilha do Cardoso. Assim, o número 20 
foi agora superado em termos de diversidade de esporos para uma mesma rizosfera. $P$. cyparisseas, no trabalho de Trufem (1988) apresentou nove diferentes espécies de fMA.

Tabela 5. Ocorrência das espécies de fMA nas rizosferas das plantas hospedeiras estudadas

\begin{tabular}{|c|c|c|c|c|c|c|c|}
\hline & 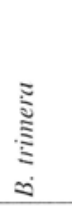 & 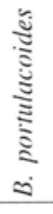 & 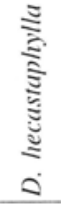 & 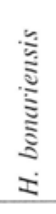 & 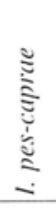 & 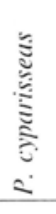 & NTPHTV \\
\hline A. laevis & + & + & - & - & - & + & 3 \\
\hline A. scrobiculata & + & - & $\cdot$ & - & + & + & 3 \\
\hline A. spinosa & + & - & - & - & - & - & 1 \\
\hline A. turbeculata & + & + & - & - & - & + & 3 \\
\hline Acaulospora sp. & + & - & - & - & - & + & 2 \\
\hline G. decipiens & + & - & - & - & - & - & 1 \\
\hline G. gigantea & + & + & + & + & + & + & 6 \\
\hline G. ramisporophora & + & - & + & + & - & + & 4 \\
\hline G. aggregatum & + & - & - & - & - & - & 1 \\
\hline G. constrictum & + & - & - & + & - & + & 3 \\
\hline G. etnicatum & + & - & - & - & - & - & 1 \\
\hline G. fasciculatum & + & - & - & - & + & + & 3 \\
\hline G. globiferum & - & - & + & + & - & - & 3 \\
\hline G. monosporum & +- & $\cdot$ & - & - & - & - & 1 \\
\hline S. sinuosum & + & - & - & - & - & - & 1 \\
\hline G. tenue & - & - & - & - & - & + & 1 \\
\hline S. calospora & + & - & + & + & + & + & 5 \\
\hline S. coralloides & + & + & + & + & - & + & 6 \\
\hline S. fulgida & + & - & - & - & - & + & 2 \\
\hline S. gilmorei & + & - & + & + & - & + & 4 \\
\hline S. gregaria & - & + & + & + & - & + & 4 \\
\hline S. persica & + & - & + & + & + & + & 5 \\
\hline S. verruscosa & - & - & + & - & - & - & 1 \\
\hline Scutellospora sp. & + & + & + & + & + & + & 6 \\
\hline NTTVPH & 20 & 6 & 10 & 10 & 7 & 17 & \\
\hline
\end{tabular}

$\mathrm{NTPHTV}=\mathrm{n}$. total de plantas hospedeiras onde o táxon de fMA foi verificado.

$\mathrm{NTTVPH}=\mathrm{n}$. total de táxons de fMA verificados em cada espécie de planta hospedeira.

Trufem (1988) constatou que, à medida que se afastava da praia indo em direção à mata, ou ao interior da Ilha do Cardoso, as espécies de Glomus e Sclerocystis iam se tornando mais abundantes e paulatinamente iam substituindo as espécies de Acaulosporo, Gigaspora e Scutellospora. Neste trabalho, verificou-se a maior abundância no número de esporos das espécies de Acaulospora e Scutellospora, o que confirmam Koske (1987) e Trufem (1990). Koske (1987) em transecto em duna, ao longo do litoral dos Estados Unidos, verificou maior abundância de esporos de Gigaspora, 
Scutellospora e Acaulospora. Glomus e Gigaspora, embora tenham ocorrido, o fizeram com poucos esporos.

Finalmente, no que se refere aos elementos químicos do solo, a tab. 6 mostra que os solos estudados são pobres em $\mathrm{P}(1 \mathrm{ug} / \mathrm{cm} 3)$ e em matéria orgânica $(0,15 \%)$, com pH levemente ácido ( $\mathrm{pH}=6,3)$, portanto, com condições ecológicas que justificam e até mesmo facilitam a ocorrência de fMA.

Tabela 6. Elementos químicos dos solos estudados.

\begin{tabular}{|c|c|c|c|c|c|c|c|c|c|c|}
\hline \multirow{2}{*}{ amostra } & \multirow{2}{*}{$\begin{array}{c}\mathrm{P} \\
\mathrm{mg} / \mathrm{cm}^{3}\end{array}$} & \multirow{2}{*}{$\begin{array}{c}\mathrm{MO} \\
\%\end{array}$} & \multirow{2}{*}{$\begin{array}{r}\mathrm{pH} \\
\mathrm{CaCl}_{2}\end{array}$} & \multicolumn{5}{|c|}{ meq. $/ 100 \mathrm{~cm}^{3}$} & \multirow{2}{*}{$\begin{array}{l}\mathrm{T} \\
\%\end{array}$} & \multirow{2}{*}{ V } \\
\hline & & & & K & $\mathrm{Ca}$ & $\mathrm{Mg}$ & $\mathrm{Al}$ & S & & \\
\hline amostra 1 & 1 & 0,2 & 6,2 & 0,07 & 0,3 & 0,7 & 0,7 & 1,1 & 1,8 & 61 \\
\hline amostra 2 & 1 & 0,1 & 6,4 & 0,12 & 0,4 & 0,5 & 0,7 & 10,0 & 1,7 & 59 \\
\hline média & 1 & 0,15 & 6,3 & 0,09 & 0,3 & 0,6 & 0,7 & 5,5 & 1,8 & 60 \\
\hline
\end{tabular}

\section{Agradecimentos}

As autoras agradecem ao Instituto Oceanográfico da Universidade de São Paulo pelo fornecimento dos dados climatológicos da época do experimento; ao Instituto Agronômico de Campinas, pela análise dos elementos químicos dos solos; à Pesquisadora Científica do Instituto de Botânica, Elisabete Aparecida Lopes, pelas identificações das plantas cujas rizosferas foram estudadas.

\section{Referências bibliográficas}

Allen, M.F. \& Boosalis, M.G. 1983. Effects of two species of vesicular arbuscular mycorrhizal fungi on drought tolerance of winter wheat. New Pltytol. 95:67-76.

Andeson, R.C., Liberta, A.E., Dickman, L.A. \& Katz., A.J. 1983. Spatial variation in VA mycorrhizal spore density. Bul. Torrey Bot. Club 10:519-525.

Cabala Rosand, P. \& Dias, R. 1986. Associações micorrízicas e a nutrição mineral das plantas. In Anais da I Reunião Brasileira sobre micorrizas. Lavras: Edições FAEPE, p. 33-59.

Daft, M.J. \& Nicolson, T.H. 1974. Vesicular-arbuscular mycorrhizas in plants colonizing coal wastes in Scotland. New Plytol. 73:1129-1139.

Gerdemann, J.W. 1968. Vesicular-arbuscular mycorrhiza and plant growth. Ann. Rev. Phytopathol. 6:397-418.

Gerdemann, J.W. \& Nicolson, T.H. 1963. Spores of mycorrhizal Endogone species extracted from soil by wet sieving and decanting. Trans. Br. mycol. Soc 46:235-244.

Giovannetti, M. 1985. Seasonal variations of vesicular-arbuscular mycorrhiza and Endogonaceous spores in a maritime sand dune. Trans. Br. mycol. Soc. 84:679-684.

Gildon, A.\& Tinker, P.B. 1983a. Interactions of VAM infections and heavy metals in plants. I. Effects of heavy metals on the development of VAM. New Phytol. 95:241-261.

Gildon, A. \& Tinker, P.B. 1983b. Interactions of VAM infections and heavy metals in plants.II The effects of infection on uptake of copper. New Phytol. 93:263-268. 
Janos, D.P. 1980. Mycorryhizae influence tropical succession. Biotropica 12:56-64.

Killham, K. \& Firestone, M.K. 1983. Vesicular-arbuscular mycorrhizal mediation of grass response to acidic and heavy metals depositions. Plant and Soil 72:39-48.

Kormanik, P.P., Bryan, W.C., Schultz, K.C.1980. Procedures and equipment for staining large numbers of plant root samples for endomycorrhizal assay. Can. J. Microbiol. 26:536-538.

Koske, R.E. 1981. A preliminary study of interactions between species of vesicular-arbuscular fungi in a sand dune. Trans. Br. mycol. Soc. 76:411-416.

Koske, R.E. 1985. Glomus aggregatum emended: a distinct taxon in the Glomus fasciculatum complex. Mycologia 77:619-630.

Koske, R.E. 1987. Distribution of VA mycorrhiazal fungi along a latitudial temperature gradient. Mycologia 79:55-68.

Koske, R.E. \& Halvorson, W.L. 1981. Ecological studies of VAM in a barrier sand dune. Can. J. Bot. 59:1413-1422.

Koske, R.E. \& Polson, E.R. 1984. Are VAM required for sand dune stabilization? Bioscience 34:420-424.

Louis, I. \& Lim, G. 1987. Spore density and root colonization of VA mycorrhizas in tropical soil. Trans. Br. mycol. Soc. 88:207-212.

McGonigle, T.P. \& Fitter, A.H. 1990. Ecological specificity of vesicular arbuscular mycorrhizal associations. Mycol. Res. 94:120-122.

Mosse, B., Stribley, D.P. \& Le Tacon, F. 1981. Ecology of mycorrhizae and mycorrhizal fungi. Adv. Microbiol. Ecol. 5:137-210.

Pfeiffer, R.M. 1981/1982. Levantamento semi- detalhado dos solos do Parque Estadual da Ilha do Cardoso, SP. Silvicultura 15/16:91-115.

Reeves, F.B., Wagner, D., Moorman, T. \& Kiel, J. 1979. The role of endomycorrhizae in revegetation pratics in the semi-arid West. I. A Comparison of incidence of mycorrhizae in severely disturbed vs. natural environmentes. Am. J. Bot. 66:6-13.

Siqueira, J.O. \& Franco, A.A. 1988. Biotecnologia do solo: Fundamentos e perspectivas. MEC. ABEAS, ESAL, FAEPE.

Spain, J.L., Sieverding, E. \& Schenck, N.C. 1989. Gigaspora ramisporophora: a new species with novel sporophores from Brazil. Mycotaxom 34:667-677.

Sylvia, D.M. \& Will, M.E. 1988. Establishment of VA mycorrhizal fungi and other microorganisms on a beach replenishment site in Florida. Appl. Environm. Microbiol. 54:348-352.

Trappe, J.M. 1977. Three new Endogonaceae: Glomus constrictum, Sclerocystis clavispora and Acaulospora scrobiculata. Mycotaxonn 6:359-366.

Trufem, S.F.B. 1988. Fungos micorrízicos vesículo-arbusculares da Illa do Cardoso, SP, Brasil. São Paulo: Universidade de São Paulo. Tese de Doutorado.

Trufem, S.F.B. 1990. Aspectos ecológicos de fungos micorrízicos vesículo-arbusculares em ecossistema de dunas do Parque Estadual da Ilha do Cardoso, SP, Brasil. II Simpósio de Ecossistemas da Costa Sul e Sudeste Brasileira: estrutura, função e manejo. São Paulo: ACIESP. vol. 3:478-487.

Trufem, S.F.B. \& Bononi, V.L.R. 1985. Micorrizas vesículo-arbusculares de culturas introduzidas em áreas de cerrado. Rickia 12:165-187.

Trufem, S.F.B., Otomo, H.S. \& Malatinszky, S.M.M. 1989. Fungos micorrízicos vesículo-arbusculares em rizosferas de plantas de dunas do Parque Estadual da Ilha do Cardoso, SP, Brasil. 1. Taxonomia. Acta bot. bras. 3:141-152. 